\title{
BIOPSIA ASPIRATIVA CON AGUJA FINA DE TIROIDES \\ Concordancia clínico patológica en pacientes del Hospital de San José 1997-2000
}

Marta Lucía Cadena Lleras, MD. ", Pilar Archila de R., MD. ", Darío Cadena Rey, MD.*

\section{Resumen}

Se hizo una revisión de 510 casos de pacientes a los que se les practicó biopsia por aspiración con aguja fina de nódulos tiroideos en el Hospital de San José, durante el período de 1997 al año 2000. La distribución por género fue: 442 mujeres y 68 hombres, con una edad promedio de 45,7 años. Las punciones fueron realizadas por los servicios de endocrinología, patología, radiología y en un importante número de casos, no se pudo determinar cuál servicio realizó la toma del examen. La sensibilidad para todas las categorías diagnósticas fue mayor de $80 \%$ y los falsos negativos menores al $20 \%$.

No se observaron diferencias estadísticamente significativas entre las diferentes categorías diagnósticas, ni cambio en la sensibilidad según el origen de la muestra. La concordancia clínico patológica no evidenció un grupo o un diagnóstico problemático. Hay una diferencia estadísticamente significativa entre las muestras enviadas de radiología y los demás servicios, ya que presentan una menor correlación, probablemente debida a fallas en el manejo y fijación del material. La frecuencia diagnóstica más alta correspondió a enfermedades benignas del tiroides. No se hallaron la especificidad, falsos positivos, valores predictivos positivos ni negativos ya que todos los casos eran positivos en el diagnóstico citológico y en la resección quirúrgica/ control clínico.

\section{Introducción}

La Biopsia Aspirativa con Aguja Fina (BACAF) de tiroides permite hacer un diagnóstico preoperatorio adecuado en el paciente con nódulo tiroideo. Para mantener la confianza en este examen es imprescindible evaluar la sensibilidad y concordancia clínico patológica del mismo. Para continuar con esta evaluación se realizó la revisión correspondiente a los años 1997-2000.

La presencia de nódulos tiroideos afecta a 4-7\% de la población adulta, predominantemente a mujeres, con un pequeño porcentaje de lesiones malignas $(5-10 \%)^{1-7}$, por lo cual es imperativo tener un diagnóstico preciso antes de proceder a realizar un tratamiento quirúrgico. La BACAF constituye un excelente método diagnóstico dentro del estudio inicial del paciente con masas del tiroides. ${ }^{1,-6,8-11}$

La técnica de la biopsia aspirativa es segura, fácil, económica y precisa. Las complicaciones son escasas

\footnotetext{
Médicos Hospital de San José

Departamento de Patología
}

y de poca gravedad, como la formación de hematoma en el sitio de punción y además, es un procedimiento ambulatorio que no requiere anestesia y es de bajo costo. $^{2,3,6,8-10,12,13}$

Las opciones en métodos diagnósticos incluyen además de la BACAF, la biopsia por Tru-Cut y por congelación. De ellos, el más fácil, seguro y con menor índice de complicaciones es la aspiración con aguja fina, y además, presenta buenos resultados con una sensibilidad del $80 \%$ según diversas fuentes de la literatura mundial. - $-3,5,6,8-10,12,14,15^{-15}$

Se requiere de patólogos con entretenimiento en citología para llegar a un diagnóstico preciso, sobre todo en algunos campos como el de las lesiones de células foliculares, donde los parámetros citológicos no son tan claros para poder diferenciar entidades malignas de benignas. ${ }^{1,5,8-10,15,16}$

Los primeros en describir la técnica de la BACAF fueron Martin y Ellis en $1930 .{ }^{17}$ A partir de ese entonces, la literatura médica reporta una gran cantidad de estudios 
en los que se observa una disminución del tratamiento quirúrgico, innecesario hasta en 50-65\%. 5, 10,17,18

Aproximadamente 75 a $85 \%$ de los exámenes patológicos tiroideos corresponden a lesiones no neoplásicas como bocio coloide, tiroiditis, lesiones foliculares benignas como adenoma y nódulo hiperplásico; 3 a $33 \%$ corresponden a extendidos inadecuados, que no permiten hacer un diagnóstico y 5 a $10 \%$ corresponden a lesiones malignas como el carcinoma papilar, medular y folicular. Entre todas las publicaciones escritas sobre la biopsia aspirativa, se observa que el grupo que presenta una mayor dificultad diagnóstica lo constituyen las lesiones de células foliculares, concretamente para distinguir adenoma de carcinoma folicular, situación que persiste aun en el estudio histopatológico. ${ }^{1-16}$ Otras circunstancias en donde se observa un mayor número de falsos negativos son el carcinoma papilar con cambio quístico, por la posible ausencia de células neoplásicas necesarias para el diagnóstico; carcinoma en presencia de tiroiditis crónica, por la escasa celularidad neoplásica en un denso fondo inflamatorio y cuando la calidad del material obtenido no es adecuada. ${ }^{18,19}$

A este respecto se abre una nueva perspectiva diagnóstica con la utilización de la inmunohistocitopatología para tratar de llegar a un diagnóstico prequirúrgico cada vez más acertado. Se han identificado moléculas como el grupo de los isómeros del CD44 y la proteína Galectina 3, que se encargan de regular varios procesos fisiopatológicos como la migración celular, el crecimiento tumoral y la interacción celular. Ambas son proteínas de membrana que se expresan en tirocitos tumorales y por tanto, su correcta identificación puede influir en la decisión terapéutica. $^{20}$

El objetivo del presente estudio fue buscar la concordancia de la citología de tiroides con el comportamiento clínico-patológico, su sensibilidad, observar si había variaciones con respecto al origen de la muestra e indagar la frecuencia diagnóstica de las lesiones del tiroides en el tiempo de estudio.

\section{Materiales y métodos}

Se diseñó un estudio observacional retrospectivo para evaluar la correlación clínico patológica de los pa- cientes a los que se les realizó biopsia aspirativa en el período comprendido entre enero de 1997 a diciembre del año 2000. Los criterios de inclusión fueron los pacientes a los que se les realizó el examen en el Hospital de San José y que tuvieron información de la historia clínica. La toma de la BACAF se realizó en los servicios de endocrinología, patología y radiología y la lectura la realizó el departamento de patología. Los extendidos fueron fijados al aire o en alcohol al $95 \%$ y se colorearon con la tinción de Wright y de Papanicolau. El tejido fue fijado en formol al $10 \%$ e incluido en bloques de parafina.

Los instrumentos utilizados fueron los informes histopatológicos, los protocolos quirúrgicos y la historia clínica con su evolución y exámenes paraclínicos, por medio de los cuales se llegó a un diagnóstico definitivo.

Los diagnósticos citológicos se dividieron en cinco categorías: bocio coloide, lesión de células foliculares (incluye adenoma, carcinoma folicular, nódulo hiperplásico), tiroiditis, carcinoma papilar y material no diagnóstico. Los datos obtenidos se analizaron con el programa estadístico SPSS y se aplicó la prueba de Chi-cuadrado.

\section{Resultados}

De los 923 pacientes a los que se les realizó el examen en el lapso del estudio, $510(55,2 \%)$ tenían historia clínica. La distribución por género fue de 442 mujeres $(86,6 \%)$ y 68 hombres $(13,4 \%)$ (Figura 1), la edad



Figura I. Distribución por géneros de la BACAF de tiroides. Hospital de San José 1997-2000 
promedio fue de 45,7 años (16-84), 45,3 para mujeres (16-84) años y 37,3 para hombres (25-84).

El servicio de endocrinología realizó 463 (50,2\%) punciones, patología 179 (19,4\%), radiología 99 $(10,7 \%)$ y los casos en que no se pudo determinar quién tomo la muestra, fueron $182(19,7 \%)$.

Se encontró que en el total de los pacientes estudiados, la sensibilidad más alta y los falsos negativos más bajos son para el carcinoma medular; la situación inversa se presenta en la tiroiditis y la lesión de células foliculares. Sin embargo no existe una diferencia estadísticamente significativa entre ellos (Tabla 1).

$\begin{aligned} & \text { Tabla 1. Sensibilidad de la BACAF de tiroides. } \\
& \text { Hospital de San José } 1997-2000\end{aligned}$
\begin{tabular}{lll} 
Diagnóstico & Sensibilidad & n \\
\hline Bocio coloide & $96,0 \%$ & 129 \\
\hline Carcinoma medular & $100,0 \%$ & 6 \\
\hline Carcinoma papilar & $90,3 \%$ & 65 \\
\hline $\begin{array}{l}\text { Lesión de células } \\
\text { foliculares }\end{array}$ & $86,5 \%$ & 58 \\
\hline Tiroiditis & $85,3 \%$ & 34
\end{tabular}

La sensibilidad más alta y los falsos negativos más bajos por servicios corresponden a patología, seguido por endocrinología y radiología, pero no existe una diferencia estadísticamente significativa entre la sensibilidad de las muestras obtenidas por los tres servicios. (Chi-cuadrado $=5,63, \mathrm{p}=\mathrm{ns}$, prueba Kruskal - Wallis) (Tabla 2).

La concordancia clínico patológica por diagnóstico citológico más alta en los cuatro años del estudio fue para el carcinoma medular, la más baja fue para la lesión de células foliculares, no hay diferencias estadísticamente significativas entre los diversos diagnósticos (Tabla 3).

La concordancia clínico patológica por servicio fue igual en patología y endocrinología y más baja en radiología.
Tabla 2. Sensibilidad por servicio de la BACAF de tiroides. Hospital de San Jose 1997-2000

\begin{tabular}{lll} 
Servicio & Sensibilidad & n \\
\hline Endocrinología & $93,1 \%$ & 192 \\
\hline Patología & $97,8 \%$ & 53 \\
\hline Radiología & $78,8 \%$ & 37
\end{tabular}

Hubo una diferencia estadísticamente significativa entre la concordancia de las muestras provenientes de los tres servicios (Chi-cuadrado $=16,3 ; \mathrm{p}=0,001$ ) (Tabla 4). Se observó una diferencia significativa entre la concordancia de endocrinología y radiología $(\mathrm{p}=0,001)$ y también es significativa la diferencia entre patología y radiología ( $p=0,001$ ). Además se aprecia una diferencia estadísticamente significativa entre los casos con concordancia y los que no tienen concordancia clínico patológica $(\mathrm{p}=0,001)$.

En la Figura 2 se observa el comportamiento de las muestras en cada servicio con relación a la concordancia, no concordancia y pacientes sin control posterior. En muestras provenientes de los todos los departamentos se observa un porcentaje similar de pacientes que no regresaron a consulta después de realizar el examen.

La frecuencia más alta de las enfermedades incluidas en el estudio fue para el bocio coloide y la más baja para la tiroiditis. En la Tabla 5 se describen estos resultados y su comportamiento anual.
Tabla 3. Concordancia clínico-patológica de la BACAF de tiroides por diagnóstico citológico. Hospital de San José $1997-2000$

\begin{tabular}{lccc} 
Diagnóstico & \multicolumn{1}{c}{$\begin{array}{c}\text { Sin } \\
\text { concordancia }\end{array}$} & $\begin{array}{c}\text { Con } \\
\text { concordancia }\end{array}$ & Total \\
\hline Bocio coloide & $11(32 \%)$ & $119(78,8 \%)$ & $151(100,0 \%)$ \\
\hline $\begin{array}{l}\text { Carcinoma } \\
\text { medular }\end{array}$ & $0(0 \%)$ & $6(100,0 \%)$ & $6(100,0 \%)$ \\
\hline $\begin{array}{l}\text { Carcinoma } \\
\text { papilar }\end{array}$ & $9(13,8 \%)$ & $56(86,2 \%)$ & $65(100,0 \%)$ \\
\hline $\begin{array}{l}\text { Lesión de } \\
\text { células foliculares }\end{array}$ & $13(22,4 \%)$ & $45(77,6 \%)$ & $58(100,0 \%)$ \\
\hline Tiroides & $5(14,7 \%)$ & $29(85,3 \%)$ & $34(100,0 \%)$ \\
\hline Total & $59(18,8 \%)$ & $255(81,2 \%)$ & $314(100,0 \%)$
\end{tabular}


Tabla 4. Concordancia clínico-patológica de la BACAF de tiroides por servicio. Hospital de San José 1997-2000

\begin{tabular}{lccr} 
Servicio & $\begin{array}{c}\text { Sin } \\
\text { concordancia }\end{array}$ & $\begin{array}{c}\text { Con } \\
\text { concordancia }\end{array}$ & Total \\
\hline Endocrinología & $29(15,1 \%)$ & $163(84,9 \%)$ & $192(100,0 \%)$ \\
\hline Patología & $8(15,1 \%)$ & $45(84,9 \%)$ & $53(100,0 \%)$ \\
\hline Radiología & $18(40,9 \%)$ & $26(59,1 \%)$ & $44(100,0 \%)$ \\
\hline Total & $55(19,1 \%)$ & $234(80,9 \%)$ & $289(100,0 \%)$
\end{tabular}

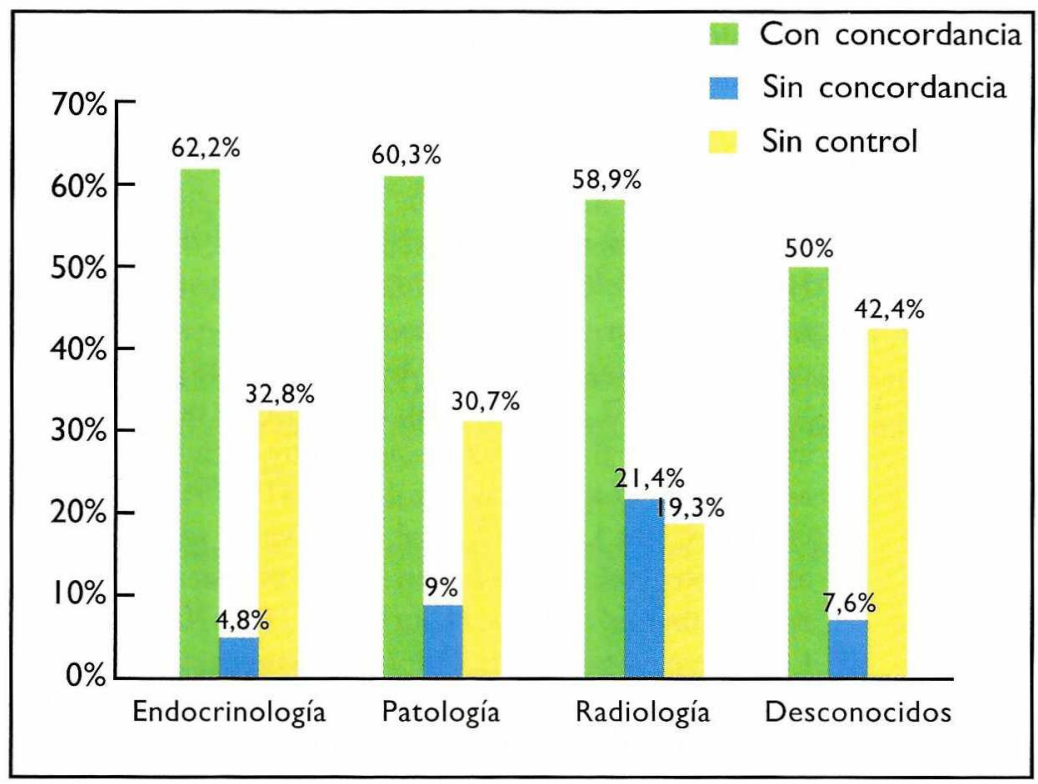

Figura 2. Concordancia clínico patológica y casos sin control por servicios. BACAF de tiroides, Hospital de San José 1997-2000

\begin{tabular}{|c|c|c|c|c|c|}
\hline Diagnóstico & 1997 & 1998 & 1999 & 2000 & Total \\
\hline Bocio coloide & $53,0 \%$ & $48,8 \%$ & $36,6 \%$ & $27,1 \%$ & $43,9 \%$ \\
\hline Carcinoma Papilar & $11,7 \%$ & $11,9 \%$ & $12,5 \%$ & $21,7 \%$ & $13,9 \%$ \\
\hline Tiroiditis & $8,0 \%$ & $5,3 \%$ & $6,8 \%$ & $5,4 \%$ & $6,4 \%$ \\
\hline $\begin{array}{l}\text { Lesión de células } \\
\text { foliculares }\end{array}$ & $7,4 \%$ & $11,3 \%$ & $12,5 \%$ & $27,1 \%$ & $12,9 \%$ \\
\hline $\begin{array}{l}\text { No } \\
\text { diagnóstico- otros }\end{array}$ & $19,9 \%$ & $22,7 \%$ & $31,6 \%$ & $18,7 \%$ & $22,9 \%$ \\
\hline
\end{tabular}

deserción del hospital, en especial en enfermedades benignas.

Las mujeres correspondieron a un $86,6 \%$ y los hombres a $13,4 \%$, con un promedio de edad de 45,3 y 37,3 respectivamente, sin presentar diferencias con la literatura. ${ }^{1-10,15,18}$

El departamento que más realizó biopsias aspirativas fue endocrinología con un 50,2\%, seguido por patología y de origen desconocido con un $19,4 \%$ y por último radiología con un $10,7 \%$. En un porcentaje importante de casos, el libro de registros de patología no contenía la información sobre quien realizó la punción.

La sensibilidad no mostró diferencias significativas entre las cinco categorías diagnósticas, así como tampoco las hubo entre los diversos orígenes de las muestras. Con respecto a la sensibilidad reportada en otras series, se observó que la nuestra se encuentra por encima de la informada en la literatura más antigua y es muy similar a la de estudios más recientes. ${ }^{1-11,13,16,18}$ La mayor sensibilidad se obtuvo para el carcinoma medular y el área de menor sensibilidad corresponde a la tiroiditis y a las lesiones de células foliculares. Estos hallazgos se corresponden con lo referido en la literatura mundial. ${ }^{1-8,12,15,16,18,19}$

En la concordancia clínico patológica entre los diferentes diagnósticos citológicos no se encontraron diferencias significativas, es decir no se evidencia algún grupo o diagnóstico que sea más problemático que otro, sin embargo la concordancia más baja fue la de las lesiones de células foliculares, que como ya se comentó es esperable, pues la biopsia aspirativa no es el método diagnóstico ideal para esta enfermedad. Es deseable implementar en un futuro cercano el uso de inmunocitohistoquímica para mejorar la concordancia. ${ }^{1-8,12,15,16,18-20}$

Respecto al origen de las muestras, se observa una diferencia estadísticamente significativa entre los servicios de endocrinología y patología respecto a radiolo- 
gía. La concordancia clínico patológica es más baja en este último servicio probablemente debido a fallas en el manejo y fijación del material obtenido en punción. En todos los servicios se observa un bajo nivel de no concordancia y como ya se comentó, hay un importante porcentaje de pacientes que no regresaron a control después de la punción.

De acuerdo con lo observado en la literatura médica global, se aprecia que en este estudio la frecuencia más alta corresponde a enfermedades benignas del tiroides, con alrededor del $22 \%$ correspondiente a muestras no diagnósticas y otros. ${ }^{1-7}$

En resumen, los resultados arrojados por este estudio confirman que la biopsia aspirativa con aguja fina constituye un excelente método diagnóstico de la patología tiroidea. Es un examen confiable pero no infalible, que debe seguir siendo utilizado en el manejo integral del paciente con nódulos tiroideos.

\section{Referencias}

1. Bibbo M. Comprehensive cytopathology. WB Saunders Company. Second Edition 2000. pag 367-384.

2. Aguilar M, Contreras A, Gavilán I, et al. Thyroid Nodules, Role of Fine Needle Aspiration and Intraoperative Frozen Section Examination. Acta Cytol 1997;41: 677-86.

3. Rojecki MT, Gharib H. Nodular thyroid disease; Evaluation and management. N Engl J Med 1995; 313: 428-36.

4. Bartolazzi A. Improving accuracy of cytology for nodular thyroid lesions. Lancet 2000; 355: 1661-2.

5. De May RM. The art and science of cytopathology. ASCP Press 1999.

6. Rosai J, Carcangiu ML, De Lellis RA. Atlas of tumor pathology: tumors of the thyroid gland. Third series, fascicle five. Washington DC: Armed Force Institute of Pathology, 1992.
7. LiVolsi V. Surgical Pathology of the thyroid. WB Saunders Company, Vol 22, 1990. pag ??

8. Bugis SP, Young JE, Archibald SD et al. Diagnostic accuracy of Fine-Needle Aspiration Biopsy versus Frozen Section in Solitary Thyroid Nodules. Am J Surg 1986; 152: 411-6.

9. Åkerman M, Tennvall J, Biörklund. Sensitivity and specificity of Fine Needle Aspiration Cytology in the diagnosis of tumors of the thyroid gland. Acta Cytol 1985; 29 : 850-5.

10. Leonard N, Melcher DH. To operate or not to operate? The value of fine needle aspiration cytology in the assessment of thyroid swellings. J Clin Pathol 1997; 50:941-3.

11. Szabolcs I, Kaszás I, Dohan O, et al. Diagnosis from thyroid aspirates. Is the cytopathologist handicapped if not fully informed about the patient?. Acta Cytol 1997; 41: 683-6.

12. Lin J, Hsueh C, Chao T, et al. Thyroid follicular neoplasms diagnosed by high-resolution ultrasonography with Fine Needle aspiration cytology. Acta Cytol 1997; 41: 687-91.

13. Hamberger B, Gharib H, Nelton LJ, et al. Fine-Needle aspiration biopsy of thyroid nodules. The impact on thyroid practice and cost care. Am J Med 1982; 73: 381-4.

14. Lin J, Huang B, Chao T, et al. Diagnosis of occult thyroid carcinoma by thyroid ulttrasonography with Fine Needle aspiration Cytology. Acta Cytol 1997; 41: 1751-5.

15. Chang H, Lin J, Chen J, et al. Correlation of fine needle aspiration cytology and frozen section biopsies in the diagnosis of thyroid nodules. J Clin Pathol 1997; 50:1005-9.

16. Löwhagen t, Sprenger E. Cytologic presentation of thyroid tumors in the aspiration biopsy smear. Acta Cytol 1974: 18: 192-7.

17. Martin HE, Ellis EB. Biopsy by needle puncture and aspiration. Ann Surg 1930; 92: 169-81.

18. Suen K, Quenville N. Fine needle aspiration biopsy of thyroid gland: a study of 304 cases. J Clin Pathol 1983; 36: 1036-45.

19. Miller JM, Hamburger JI, Kini S. The needle biopsy diagnosis of papillary thyroid carcinoma. Cancer 1981; 48:989-93.

20. Bartolazzi A, Gasbarri A, Papotti M, et al. Application of an immunodiagnostic method for improving preoperative diagnosis of nodular thyroid lesions. Lancet 2001; 357: 1644-50. 